\title{
Image restoration by partial differential equations
}

\author{
Mirjana Štrboja
}

Department of Mathematics and Informatics, University of Novi Sad Trg D. Obradovica 4, 21000 Novi Sad, Serbia

e-mail: mirjanas@im.ns.ac.yu

\begin{abstract}
It is given a short overview on models for image restoration where an image is embedded in an evolution process. The original image is transformed through a process that can be represented in form of second-order partial differential equation. It is considered a general form of PDE-based methods for restoration. Models which are generalizations of the heat equation are given.
\end{abstract}

Keywords: Image restoration, heat equation, nonlinear diffusion, Perona and Malik model.

\section{Introduction}

We give a short overview how PDEs methods can be applied in the restoration of one image $([1,3,5,9])$. The idea is to superimpose a regular grid on an analogue image and to assign a digital number to each square of the grid. Each square is called a pixel. Value of pixel is the gray-level or brightness. To describe a pixel may need several channels. Three channels are necessary for a color image. We will consider only gray-scale images with one channel. A restored image can be seen as a version of the initial image at a special scale. An image $u$ is embedded in an evolution process, denote by $u(t, \cdot)$. At time $t=0$, $u(0, \cdot)=u_{0}(\cdot)$ is the original image. The original image is then transformed a process than can be written $\frac{\partial u}{\partial t}(t, x)+F\left(x, u(t, x), \nabla u(t, x), \nabla^{2} u(t, x)\right)=0$ in $\Omega$. Some possibilities for $F$ to restore an image are considered in [1]. In Section 2 it is presented a general form of PDE for image restoration and there is given a connection with Gaussian linear filtering. The basic PDE in image restoration is the heat equation. Because of its oversmoothing property (edges get smeared), it is necessary to introduce some nonlinearity. We then consider the model $([1,9])$

$$
\frac{\partial u}{\partial t}=\operatorname{div}\left(c\left(|\nabla u|^{2}\right) \nabla u\right)
$$


where we choose the function $c$ that equation remains parabolic. We would like to have $c(s) \approx 1 / \sqrt{s}$ as $s \rightarrow \infty$, because we want to preserve the discontinuities, see [1]. Because of this behavior, it is not possible to apply general results for parabolic equations theory. The framework for study this equation is the nonlinear semigroup theory $([1,2,4])$. In Section 3 it is introduced some regularization. Catte et al. [3] proposed to solve the following partial differential equation instead (1):

$$
\frac{\partial u}{\partial t}(t, x)=\operatorname{div}\left(c\left(\left|\left(\nabla G_{\sigma} * u\right)(t, x)\right|^{2}\right) \nabla u(t, x)\right) .
$$

\section{General form of PDE-Based Method}

Some divergence operators can be decomposed using the tangent and normal directions to the isophote lines (lines along which the intensity is constant), see [1]. For each point $x$ where $|\nabla u| \neq 0$ we can define the vectors $N=\frac{\nabla u}{|\nabla u|}$ and $T$ with $T \cdot N=0,|T|=1$. For the first and second partial derivatives of $u$ we use the usual notation $u_{x_{1}}, u_{x_{2}}, u_{x_{1} x_{1}, \ldots}$ We denote by $u_{N N}$ and $u_{T T}$ the second derivatives of $u$ in the $T$-direction and $N$-direction, respectively:

$$
\begin{aligned}
& u_{T T}=T^{t} \nabla^{2} u T=\frac{1}{|\nabla u|^{2}}\left(u_{x}^{2} u_{y y}+u_{y}^{2} u_{x x}-2 u_{x} u_{y} u_{x y}\right), \\
& u_{N N}=N^{t} \nabla^{2} u N=\frac{1}{|\nabla u|^{2}}\left(u_{x}^{2} u_{x x}+u_{y}^{2} u_{y y}+2 u_{x} u_{y} u_{x y}\right) .
\end{aligned}
$$

$\nabla u$ and $\nabla^{2} u$ are respectively the gradient and the Hessian matrix of $u$ with respect to the space variable $x$.

PDE-methods for restoration can be formally written in the following general form:

$$
\left\{\begin{array}{l}
\frac{\partial u}{\partial t}(t, x)+F\left(x, u(t, x), \nabla u(t, x), \nabla^{2} u(t, x)\right)=0 \text { in }(0, T) \times \Omega, \\
\frac{\partial u}{\partial N}(t, x)=0 \text { on }(0, T) \times \partial \Omega \text { (Neumann boundary condition), } \\
u(0, x)=u_{0}(x) \text { (initial condition), }
\end{array}\right.
$$

where $u(t, x)$ is the restored version of the initial degraded image $u_{0}(x)$. The idea is to construct a family of functions (i.e. images) $\{u(t, x)\}_{t>0}$ representing successive version of $u_{0}(x)$. As $t$ increases $u(t, x)$ changes into a more and more simplified image. We would like to attain two goals. The first is that $u(t, x)$ should represent a smooth version of $u_{0}(x)$ where the noise has been removed. The second is to be able to preserve some features such as edges, corners, and T-junctions, which may be viewed as singularitis.

The basic PDE in image restoration is the heat equation:

$$
\left\{\begin{array}{l}
\frac{\partial u}{\partial t}(t, x)-\Delta u(t, x)=0, t \geq 0, x \in \mathbb{R}^{2}, \\
u(0, x)=u_{0}(x)
\end{array}\right.
$$


We consider that $u_{0}(x)$ is primarily defined on the square $[0,1]^{2}$. We extend it by symmetry to $C=[-1,1]^{2}$ and then in all of $\mathbb{R}^{2}$ by periodicity. This way of extending $u_{0}(x)$ is classical in image processing. If $u_{0}(x)$ extended in this way satisfies in addition $\int_{C}\left|u_{0}(x)\right| d x<+\infty$, we will say that $u_{0} \in L_{\#}^{1}(C)$ (see [1]).

Solving (3) is equivalent to carrying out a Gaussian linear filtering, which was widely used in signal processing. If $u_{0} \in L_{\#}^{1}(C)$, then the explicit solution of (3) is given by

$$
u(t, x)=\int_{\mathbb{R}^{2}} G_{\sqrt{2 t}}(x-y) u_{0}(y) d y=\left(G_{\sqrt{2 t}} * u_{0}\right)(x),
$$

where $G_{\sigma}(x)$ denotes the two-dimensional Gaussian kernel

$$
G_{\sigma}(x)=\frac{1}{2 \pi \sigma} e^{-\frac{|x|^{2}}{2 \sigma^{2}}}
$$

In [1] the authors introduce directions $N=\frac{\nabla u}{|\nabla u|}$ i $T$ with $T \cdot N=0,|T|=1$ and then is $\Delta u=u_{N N}+u_{T T}$. The isotropy means that the diffusion is equivalent in two directions.

Proposition 1 Let $u_{0}$ be in $L_{\#}^{1}(C)$ and define $u(t, x)$ by (4). Than for all $t>0$ and $x \in \mathbb{R}^{2}, u(t, x)$ satisfies the heat equation with initial value $u_{0}$ :

$$
\begin{aligned}
& \frac{\partial u}{\partial t}(t, x)=\Delta u(t, x) \text { and } \lim _{t \rightarrow 0} \int_{C}\left|u(t, x)-u_{0}(x)\right| d x=0 \\
& u(t, \cdot) \in L_{\#}^{1}(C) \text { and } u \in C^{\infty}\left((0, T) \times \mathbb{R}^{2}\right) \text { for all } T>0 .
\end{aligned}
$$

Moreover, if $t_{1}$ is any positive real number, there exists a constant $c\left(t_{1}\right)$ such that for $t \in\left[t_{1},+\infty\right)$,

$$
\sup _{x \in \mathbb{R}^{2}}|u(t, x)| \leq c\left(t_{1}\right)\left|u_{0}\right|_{L_{\#}^{1}(C)}
$$

If $u_{0} \in L_{\#}^{\infty}(C)$, than we have a maximum principle

$$
\inf _{x \in \mathbb{R}^{2}} u_{0}(x) \leq u(t, x) \leq \sup _{x \in \mathbb{R}^{2}} u_{0}(x)
$$

Here $u(t, x)$ given by (4), is the unique solution of the heat equation satisfying conditions (6) and (7).

The heat equation has been (and is) successfully applied in image processing but it has some drawback. It is too smoothing and because of that edges can be lost or severely blurred.

We will consider models that are generalizations of the heat equation. The domain image will be a bounded open set $\Omega$ of $\mathbb{R}^{2}$. The following equation is 
initially proposed by Perona and Malik [9]:

$$
\left\{\begin{array}{l}
\frac{\partial u}{\partial t}=\operatorname{div}\left(c\left(|\nabla u|^{2}\right) \nabla u\right) \quad \text { in } \Omega \times(0, T), \\
\frac{\partial u}{\partial N}=0 \text { on } \partial \Omega \times(0, T), \\
u(0, x)=u_{0}(x) \text { in } \Omega
\end{array}\right.
$$

where $c:[0, \infty) \rightarrow(0, \infty)$. If we choose $c \equiv 1$, then it is reduced on the heat equation. If we assume that $c(s)$ is a decreasing function satisfying $c(0)=1$ and $\lim _{s \rightarrow \infty} c(s)=0$, then:

i) Inside the regions where the magnitude of the gradient of $u$ is weak, equation (8) acts like the heat equation, resulting in isotropic smoothing.

ii) Near the region's boundaries where the magnitude of the gradient is large, the regularization is "stopped" and the edges are preserved.

We choose $c$ so that equation remains parabolic. We would like to have $c(s) \approx 1 / \sqrt{s}$ as $s \rightarrow \infty$, because we want to preserve the discontinuities (see [1]). Because of this behavior, it is not possible to apply general results from parabolic equations theory.

The assumptions imposed on $c(s)$ are

$$
\left\{\begin{array}{c}
c:[0, \infty) \rightarrow(0, \infty) \text { decreasing, } \\
c(0)=1, c(s) \approx \frac{1}{\sqrt{s}} \text { as } s \rightarrow \infty, \\
b(s)=c(s)+2 c^{\prime}(s)>0 .
\end{array}\right.
$$

Framework to study this equation is nonlinear semigroup theory (see $[1,2,4])$. In [1] is given theorem which establish the existence and uniqueness of a solution.

Example of $c(s)$ satisfying (9) is $c(s)=\frac{1}{\sqrt{1+s}}$.

Now we represent the Perona and Malik model [9]. We consider equations that can behave locally as inverse heat equations. Perona and Malik in [9] consider follow choices for function $c$ in equation (1):

$$
c(s)=\frac{1}{1+s / k} \text { or } c(s)=e^{-s / k}
$$

where $k$ is constant. The divergence term in (1) can be written as

$$
\operatorname{div}\left(c\left(|\nabla u(t, x)|^{2}\right) \nabla u(t, x)\right)=c\left(|\nabla u(t, x)|^{2}\right) u_{T T}+b\left(|\nabla u(t, x)|^{2}\right) u_{N N},
$$

where we denote by $T$ and $N$ the tangent and normal directions to the isophotes and function $b$ depends on $c$. With choices of $c$ like (10), the function $b$ may become negative. Solution should consist of regions with "low" gradients separated by points of discontinuity where the gradient is infinitive (see [5]). The 
notion of solution must be understood in a measure sense, and it is still an open problem. Another possibility is to introduce some regularization. Catte et al. [3] proposed to solve the following partial differential equation instead (1):

$$
\frac{\partial u}{\partial t}(t, x)=\operatorname{div}\left(c\left(\left|\left(\nabla G_{\sigma} * u\right)(t, x)\right|^{2}\right) \nabla u(t, x)\right) .
$$

This equation is well posed, in Theorem 2 is proved the existence and uniqueness of a solutions in the distributional sense.

The following equation is the general 2-D Perona and Malik [9].

$$
\left\{\begin{aligned}
\frac{\partial u}{\partial t}(t, x) & =\operatorname{div}\left(c\left(|\nabla u(t, x)|^{2}\right) \nabla u(t, x)\right), \\
u(0, x) & =u_{0}(x)
\end{aligned}\right.
$$

where $c:[0, \infty) \rightarrow(0, \infty)$ is a smooth decreasing function. Equation (11) can be rewritten as

$$
\left\{\begin{aligned}
\frac{\partial u}{\partial t}(t, x) & =c\left(|\nabla u(t, x)|^{2}\right) u_{T T}+b\left(|\nabla u(t, x)|^{2}\right) u_{N N} \\
u(0, x) & =u_{0}(x) .
\end{aligned}\right.
$$

Following intuition from the 1-D case and to sharpen edges, authors of [1] impose that (11) is backward in the normal direction $N$, i.e.,

$$
b(s)=c(s)+2 s c^{\prime}(s)<0 \text { for large } s \geq K
$$

where $K$ is a given threshold. If we want to smooth homogeneous regions, we can assume

$$
c(0)=b(0)=1
$$

which implies that (11) acts like the heat equation for small gradients.

Hardly anything can be said about the existence of solution for (11) with $b$ satisfying (12). Kichenassamy [5] proved the following theorem:

\section{Theorem 1 Let us suppose that:}

i) There exists a constant $K>0$ such that $b(s)>0$ for $s<K^{2}$ and $b(s)<0$ for $s>K^{2}$

ii) Both $c(s)$ and $b(s)$ tend to zero as $s \rightarrow \infty$.

iii) (11) has a solution $u(t, x)$ satisfying $K_{1} \leq u_{x}(t, x) \leq K_{2}$ for all $x \in[A, B]$ and all $t \in[0, T]$, for some $A, B i K_{1}>K$.

Then $u(t, x)$ is infinitely differentiable at $t=0$ and for all $x \in(A, B)$. Therefore, if the initial image is not infinitely differentiable, there is no weak solution.

It follows from [5] that "solution" must consist of regions in which it has a gradient less than $K$ in absolute value separated by points of discontinuity where the gradient is infinite. Thus, the notion of solution must be understood in the measure sense. A typical example for $c(s)$ is $c(s)=\frac{1}{1+s / K}$. 


\section{Regularization of Perona and Malik model}

In this section will introduce a regularization that makes the problem well posed. This method was followed by Catte et al. [3]. The idea is to substitute in diffusion coefficient $c\left(|\nabla u|^{2}\right)$ the gradient of the image $\nabla u$ by a smooth version of it $G_{\sigma} * \nabla u$ is a smoothing kernel, for example, the Gaussian one (5). Since $G_{\sigma} * \nabla u=\nabla\left(G_{\sigma} * u\right)=\nabla G_{\sigma} * u$, model is

$$
\left\{\begin{aligned}
\frac{\partial u}{\partial t}(t, x) & =\operatorname{div}\left(c\left(\left|\left(\nabla G_{\sigma} * u\right)(t, x)\right|^{2}\right) \nabla u(t, x)\right), \\
u(0, x) & =u_{0}(x) .
\end{aligned}\right.
$$

Next theorem from [3] establish that (13) is well posed. Let us note $\Omega=$ $(0,1) \times(0,1)$ and $g(s)=c\left(s^{2}\right)$.

Theorem 2 Let $g:[0, \infty) \rightarrow(0, \infty)$ be smooth, decreasing with $g(0)=1$, $\lim _{s \rightarrow+\infty} g(s)=0$ and $s \rightarrow g(\sqrt{s})$ smooth. If $u_{0} \in L^{2}(\Omega)$, then there exists a unique function $u(t, x) \in C\left([0, T] ; L^{2}(\Omega)\right) \cap L^{2}\left((0, T) ; W^{1,2}(\Omega)\right)$ satisfying in the distributional sense

$$
\left\{\begin{array}{l}
\frac{\partial u}{\partial t}(t, x)-\operatorname{div}\left(c\left(\left|\left(\nabla G_{\sigma} * u\right)(t, x)\right|^{2}\right) \nabla u(t, x)\right)=0 \text { on }(0, T) \times \Omega, \\
\frac{\partial u}{\partial N}(t, x)=0 \text { on }(0, T) \times \partial \Omega \\
u(0, x)=u_{0}(x) .
\end{array}\right.
$$

Moreover, $|u|_{L^{\infty}\left((0, T) ; L^{2}(\Omega)\right)} \leq\left|u_{0}\right|_{L^{2}(\Omega)}$ and $u \in C^{\infty}((0, T) \times \bar{\Omega})$.

Remark Let $u_{0} \in L^{2}(\Omega)$. We construct a sequence $u^{n}$ by solving the iterative scheme

$$
\left\{\begin{array}{l}
\frac{\partial u^{n}}{\partial t}(t, x)-\operatorname{div}\left(g\left(\left|\left(\nabla G_{\sigma} * u^{n}\right)(t, x)\right|^{2}\right) \nabla u^{n+1}(t, x)\right)=0 \text { a.e. on }(0, T) \times \Omega, \\
\frac{\partial u^{n+1}}{\partial N}(t, x)=0 \text { a.e. on }(0, T) \times \partial \Omega \\
u^{n+1}(0, x)=u_{0}(x) .
\end{array}\right.
$$

It is proven in [3] that $u^{n}$ converges in $C\left([0, T] ; L^{2}(\Omega)\right)$ to the unique solution of (14).

\section{Conclusion}

In this paper we give a short overview how PDEs methods can be applied in the restoration of one image. Further research should concern pseudo-analysis $([6,7,8])$ and its applications on this problem.

Acknowledgement. This paper has been supported by the project MNZŽSS144012 . 


\section{References}

[1] G. Aubert, P. Kornprobst, "Mathematical Problems in Image Processing", Springer-Verlag, 2002.

[2] H. Breyis, "Opérateurs Maximaux Monotones et Semi-Groupes de Contractions dans les Espaces de Hilbert", North-Holland Publishing Comp, Amsterdam-London, 1973.

[3] F. Catte, P.L. Lions, J.M. Morel, T. Coll, "Image selective smoothing and edge detection by nonlinear diffusion", SIAM Journal of Numerical Analysis, 29(1):182-193, 1992.

[4] T. Cazenave, A. Haraux, "Introduction aux Problemes d'Evolution SemiLinéares",(Introduction to Semilinear Evolution Problems), Mathématiques \& Apllications, Ellipses, 1990.

[5] S. Kichenassamy, "The Perona-Malik paradoks", SIAM Journal of Applied Mathematics, 57(5):1328-1342, 1997.

[6] V. P. Maslov, S.N. Samborskij (eds.), "Idempotent Analysis", Advances in Soviet Mathematics 13, Providence, Rhode Island, Amer. Math. Soc.., 1992.

[7] E. Pap, "Applications of decomposable measures", in Handbook Mathematics of Fuzzy Sets-Logic, Topology and Measure Theory (Ed. U. Höhle, R.S. Rodabaugh), Kluwer Academic Publishers, 1999, 675-700.

[8] E. Pap, "Null-Additive Set Functions", Kluwer Academic Publishers, Dordrecht- Boston-London, 1995.

[9] P. Peron, J. Malik, "Scale-space and edge detection using anisotropic diffusion", IEEE Transactions on Pattern Analysis and Machine Intelligence, 12(7): 629-639, 1990. 